\title{
A CONLEY INDEX CALCULATION
}

\author{
E. N. DANCER
}

(Received 7 April 2009)

\begin{abstract}
We discuss a Conley index calculation which is of importance in population models with large interaction. In particular, we prove that a certain Conley index is trivial.
\end{abstract}

2000 Mathematics subject classification: primary 35K55, 35K57.

Keywords and phrases: Conley index, connecting orbits.

\section{Introduction}

We calculate the Conley index of a suitable isolated invariant set $T$ for the semiflow $\dot{x}=H(x)$ on a Banach space $E$. Here $T$ consists of two hyperbolic stationary points $u_{0}$ and $u_{1}$ and a single orbit joining $u_{0}$ to $u_{1}$ where the unstable manifold of $u_{0}$ intersects the stable manifold of $u_{1}$ transversally. We prove that the Conley index is trivial, under natural hypotheses.

We briefly explain some of the terms in the previous paragraph; more details can be found in [11]. A semiflow need only be defined for $t \geq 0$. A stationary point $x_{0}$ is said to be hyperbolic if the linearization $H^{\prime}\left(x_{0}\right)$ exists and none of the spectrum of $H^{\prime}\left(x_{0}\right)$ lies on the imaginary axis. By a connecting orbit joining $u_{0}$ to $u_{1}$, we mean a solution $u(t)$ of $\dot{x}=H(x)$ such that $u(t) \rightarrow u_{0}$ in $E$ as $t \rightarrow-\infty$ and $u(t) \rightarrow u_{1}$ in $E$ as $t \rightarrow \infty$. The stable manifold of $u_{1}$ is defined locally to be the points of $E$ near $u_{1}$ which lie on solutions of $\dot{x}=H(x)$ which tend to $u_{1}$ as $t \rightarrow \infty$ and is then extended globally. The unstable manifold of $u_{0}$ is defined similarly (though there are some complications as we only have a semiflow). The definition of transversality is a slight variant of the usual definition of transversality in differential topology and is discussed in [11]. (The usual definition needs to be changed slightly because, if $u(t)$ is a connecting orbit, $u^{\prime}(t)$ always lies in the tangent spaces of both the stable and unstable manifold at $u(t)$.)

Note that the Conley index is a topological invariant for compact isolated invariant sets of dynamical systems. It has been used extensively to study the bounded solutions

(C) 2009 Australian Mathematical Publishing Association Inc. 0004-9727/2009 \$16.00 
of such equations; see [6] for the case of flows on finite-dimensional spaces and [15] for the infinite-dimensional case (for a certain class of equations). The basic notions of the Conley index are defined in [15]. In particular, the Conley index of $T, \hat{h}(T)$, is defined to be the homotopy type of $[S / \tilde{E}]$ (with base point) where $S$ is a rather nice neighbourhood of $T$ (an isolating block in the notation of [15]). Here $\tilde{E}$ is the subset of $S$ where the semiflow of $H$ exits $S$ and [ ] denotes the homotopy type (with base point $\tilde{E}$ ).

The reason why the above result is of interest is the following. As noted in [8], the above result implies that the connection index is nontrivial. The connection index is a 'count' of the connecting orbits from $u_{0}$ to $u_{1}$ in $T$ and is defined in [14]. In particular, under deformations of the equation the connection index stays the same under quite general perturbations and thus we can frequently prove connections from $u_{0}$ to $u_{1}$ persist. Indeed, this was the motivation for the present paper. In [7] we considered a population model,

$$
\begin{gathered}
u_{t}=\Delta u+f(u)-k u v, \\
v_{t}=\Delta v+g(v)-\alpha k u v
\end{gathered}
$$

on a bounded smooth domain $\Omega$ with homogeneous Dirichlet boundary conditions on $\partial \Omega$. Here $\alpha, k>0, f, g$ are $C^{1}, f(0)=0$, and $g(0)=0$. This is a competing species model with diffusion which has been extensively studied (see [7, 9, 10], for example). Because $u$ and $v$ are populations, it is natural to consider only solutions with $u, v$ nonnegative. It is proved in [9] that for large $k$, there is a natural limit problem,

$$
\begin{array}{ll}
\dot{\omega}=\Delta \omega+h(\omega) & \text { in } \Omega, \\
\omega=0 & \text { on } \partial \Omega,
\end{array}
$$

where $h(y)=\alpha f\left(\alpha^{-1} y^{+}\right)-g\left(y^{-}\right)$and where we allow $\omega$ to change sign. (We recover $u, v$ from $\omega$ by taking $u=\alpha^{-1} \omega^{+}, v=-\omega^{-}$.) There are actually two separate limit problems for this equation. The other limit problem is for small solutions and large $k$. We do not discuss this one here; it is covered in [8]. It is proved in [8] that Conley indices of (1.1) for large $k$ (in $\{(u, v) \in X \times X \mid u, v$ are nonnegative $\}$ ) for an appropriate Banach space $X$ are equal to an appropriate corresponding Conley index of (1.2) on all of $X$ at least up to cohomology. This enables us to prove the existence of connecting orbits between stationary solutions of (1.1) corresponding to two nontrivial solutions of $\omega_{1}, \omega_{2}$ of (1.2) for large $k$ if we prove that the connection index of an orbit $T$ joining $\omega_{1}$ and $\omega_{2}$ is nontrivial (for the semiflow corresponding to (1.2)). In this way our claim will complete the proof of Corollary 2.7(b) in [8]. Note that our problem is really a singular perturbation problem with small parameter $k^{-1}$ and we have additional difficulties since $u$ and $v$ are required to be nonnegative while $w$ may change sign. (Thus the two Conley indices are on different spaces.) Hence, it seems difficult to use the implicit function theorem to prove the existence of the connection. Note that we also have considerable technical difficulties because $h$ is not $C^{1}$. 


\section{Main result}

We now state our main result. We will not state it in the most general form, but the result covers our application. We consider the problem

$$
\begin{array}{ll}
\dot{u}=\Delta u+h(u) & \text { in } \Omega, \\
u=0 & \text { on } \partial \Omega,
\end{array}
$$

with $h$ as before. Because our $h$ is not $C^{1}$ at zero, there will be technical difficulties which we will have to overcome. (It is Lipschitz on compact sets and $C^{1}$ except at zero.) It is convenient to modify $h$ for large $y$ so that $\left|y h^{\prime}(y)\right|+|h(y)|$ is bounded on $\mathbb{R}$ and $y h(y)<0$ for $|y|$ large. Using maximum principles, it follows easily that there is an $L^{\infty}$ bound for both stationary solutions and connecting orbits of (1.1).

Assumption A. Assume that:

(i) $u_{0}$ and $u_{1}$ are hyperbolic stationary solutions of (1.2);

(ii) there is a connecting orbit $T$ joining $u_{0}$ to $u_{1}$;

(iii) Morse index of $u_{1}+1=$ Morse index of $u_{0}$; and

(iv) the unstable manifold of $u_{0}$ intersects the stable manifold of $u_{1}$ transversally on $T$.

We need to explain this assumption a little, including why the various terms make sense. Firstly, it is known by [3] that, if $\omega$ is a nontrivial solution of (1.2), then $\omega$ only vanishes on a set of measure zero and hence $h$ is differentiable (in fact strictly differentiable in the sense of [4]) at $\omega$ in appropriate spaces, and so the linearization makes sense. As we see below, this is enough smoothness to prove the existence of the stable and unstable manifolds at $u_{0}$ and $u_{1}$. If $z(t, x)$ is a connecting orbit joining $u_{0}$ and $u_{1}$, it follows from [5] (see Lemma 3.2 below) that, for each $t,\{x \in \Omega \mid z(t, x)=$ $0\}$ has measure zero in $\Omega$. Hence, the linearization at $z(t, x)$ is defined and hence transversality makes sense. The strategy of our proof will be to show that if we take an isolating neighbourhood $N$ of $T \cup\left\{u_{0}, u_{1}\right\}$, then the Conley index of the semiflow of (1.2) on $T$ is the same as that of the 'semiflow' of (1.2) on the finite-dimensional space $P_{n} X$ where $h$ is replaced by $P_{n} h\left(P_{n}\right)$ for large $n$ and where $P_{n}$ is the orthogonal (in $L^{2}(\Omega)$ ) projection onto the space spanned by the eigenfunctions corresponding to the first $n$ distinct eigenvalues of $-\Delta$ for Dirichlet boundary conditions on $\Omega$. Here we prove (and this is the technical part) that our structure, including a single transversal intersection of the stable and unstable manifolds, is preserved and hence we have reduced to the finite-dimensional case. We then smooth carefully and use a result of McCord [14]. (Unfortunately, it does not appear to be easy to generalize McCord's proof to infinite dimensions.) We now state our result precisely and give the tedious details. Note that we could easily state a rather more general abstract result if the mapping $h$ is $C^{1}$.

THEOREM 2.1. Assume that $\Omega$ is a smooth bounded domain in $\mathbb{R}^{N}, h$ is as above and that Assumption A holds. If $Y$ is an isolating neighbourhood of $\left\{u_{0}, u_{1}, T\right\}$ for the 
semiflow of (1.2) on the fractional power space $X^{\alpha}$ where $0<\alpha<1$ and $X=L^{2}(\Omega)$, then $\hat{h}(Y, S(t))$ is trivial.

REMARK 2.2. There is nothing special about $L^{2}(\Omega)$. By using [8, Lemma 2.1], we can replace $L^{2}(\Omega)$ by $L^{p}(\Omega)$ where $1<p<\infty$ (at least for the cohomology of the Conley index, which is sufficient for nearly all applications). Note that fractional power spaces are defined in [11].

The author feels that it should be possible to give a more natural direct proof of the theorem, at least in the $C^{1}$ case.

LEMMA 2.3.

(i) If $\phi \in L^{2}(\Omega), P_{n} \phi \rightarrow \phi$ in $L^{2}(\Omega)$ as $n \rightarrow \infty$.

(ii) If $\phi \in \dot{W}^{1,2}(\Omega) \cap W^{2,2}(\Omega), P_{n} \phi \rightarrow \phi$ in $W^{2,2}(\Omega)$ as $n \rightarrow \infty$.

PROOF. Part (i) follows since the range of the $P_{n}$ s together forms an orthonormal basis for $L^{2}(\Omega)$, then (ii) follows from (i) since the $P_{n}$ s commute with the Laplacian.

REMARK 2.4. We can use interpolation to obtain convergence in many other spaces, in particular in $\dot{W}^{1,2}(\Omega)$.

Proof of TheOrem 2.1. We first consider the stationary solution $u_{0}$. Since $u_{0}$ is nontrivial, a classical result [3] implies that $u_{0}$ only vanishes on a set of measure zero. It then follows as in [7, p. 248] that the map $\omega \rightarrow h(\omega)$ is strictly differentiable at $u_{0}$ in the sense of [4] as a map of $L^{p}(\Omega)$ into $L^{q}(\Omega)$ if $p>q \geq 1$ (see also Lemma 3.2 below). It follows easily by regularity and compactness of the Laplacian that the map $\omega \rightarrow(-\Delta)^{-1} h(\omega)$ is a completely continuous mapping of $L^{p}(\Omega)$ into itself if $1<p<\infty$ and is strictly differentiable at $u_{0}$. It follows easily from this that for $n$ large, the problem $-\Delta Y=P_{n} h\left(P_{n} Y\right)$ has a unique solution $u_{0}^{n}$ in $h\left(P_{n}\right)$ close to $u_{0}$ in $L^{2}(\Omega)$. This is straightforward (by the contraction mapping theorem). We do not give the details because similar but more complicated arguments appear below. Moreover, for large $n$, the operator $-\Delta \tilde{h}-P_{n} h^{\prime}\left(u_{0}^{n}\right) P_{n} \tilde{h}=\lambda \tilde{h}$ on $R\left(P_{n}\right)$ has the same number of negative eigenvalues as $-\Delta-h^{\prime}\left(u_{0}\right) I$ on $\dot{W}^{1,2}(\Omega)$. (Note that $u_{0}^{n}$ is a finite linear combination of eigenfunctions and hence is analytic in $\Omega$. Thus it only vanishes on a set of measure zero.) This follows easily from standard spectral results if we note that $h^{\prime}\left(u_{0}^{n}\right)$ converges pointwise to $h^{\prime}\left(u_{0}\right)$ almost everywhere and is uniformly bounded. (Thus the convergence is strong in $L^{p}$ for all finite $p$.)

We now consider the parabolic equation

$$
\begin{array}{ll}
\dot{u}=\Delta u+h(u) & \text { on } \Omega, \\
u=0 & \text { on } \partial \Omega .
\end{array}
$$

Since $h$ is strictly differentiable at $u_{0}$ as a map of $L^{p}(\Omega)$ into $L^{q}(\Omega)$ for $p>q$, it is easy to combine with regularity properties of the Laplacian to prove the existence of a local unstable manifold $W^{u}$ for $u_{0}$ in the sense of [11]. (This is an easy 
modification of [11, proof of Theorem 6.1.2].) The strict differentiability guarantees a small Lipschitz condition near the stationary point for the remainder term. The same argument guarantees the existence of a stable invariant manifold near $u_{1}$. Similar arguments also guarantee the existence of local stable manifolds $W^{s, n}$ near $u_{0}^{n}$ and these are parameterized over the unstable space of $u_{0}$ uniformly in $n$ (by examining the contraction mapping proof of the existence of the local manifold theorem in [11, p. 112]). Similar remarks hold for the unstable manifold of $u_{1}^{n}$ which is denoted by $W^{u, n}$. In both cases, as examination of the proof shows strictly differentiability at the critical point. As in [11, pp. 154-156] there corresponds a global unstable manifold that exists and is in fact a $C^{1}$ manifold (which ensures that it has a tangent space). Note that this uses the variational structure because our scalar problem has energy $\int_{\Omega}\left(\frac{1}{2}(\nabla u)^{2}+H(u)\right)$ where $H^{\prime}=h$ (with a similar result for the projected problem).

There are several technical issues in the above. Firstly, the proof of the existence of the global unstable manifold requires backward uniqueness for the equation $\dot{u}+\Delta u=$ $h(u)$. Since $h$ is globally Lipschitz, this is true if we show that, if $z$ is a nontrivial solution of $\dot{z}+\Delta z=a(x, t) z$ on $\Omega \times R$, if $z$ satisfying the boundary condition, if $a$ is bounded on $\Omega \times \mathbb{R}$ and if $z(x, t)=0$ if $x \in \Omega, t \geq 0$, then $z$ vanishes identically. This follows from [2, Theorem 2.1].

Secondly, we need to know that if $\delta>0$ is small, then the time map $u \rightarrow T(\delta) u$ is $C^{1}$ along an unstable manifold. This follows from [11, proof of Theorem 3.4.4] and the strict differentiability of $h$ at any point of the unstable manifold. (For this, we also need to know that any nontrivial solution of $\dot{u}=\Delta u+h(u)$ on $\Omega \times \mathbb{R}$ satisfying the boundary conditions has the property that $\{x \in \Omega \mid u(x, t)=0\}$ has measure zero in $\mathbb{R}^{N}$ for each $t \in \mathbb{R}$. This follows from a slight variant of the main theorem in [5]. The details appear in Lemma 3.1 below.)

The strict differentiability of the map $x \rightarrow h(x)$ as a map of $C\left([0, T], X^{\alpha}\right)$ into $C([0, T], X)$ at a solution is similar to the proof of a result in $[7$, p. 248]. Since this is technical, we defer it to Lemma 3.2 below. The same argument proves that the local stable manifold is strictly differentiable at any point $u(x, t)$ such that $\{x \in \Omega \mid u(x, t)=0\}$ has measure zero for each $t \in R$. In particular, this applies at any point of $W^{u}(a) \cap W_{\mathrm{loc}}^{s}(b)$ where $a$ and $b$ are critical points. Thus in particular $W_{\text {loc }}^{s}(b)$ has tangent space at such points. Hence, we can define that $W^{u}(a)$ and $W^{s}(b)$ intersect transversally if $W^{u}(a)$ and $W_{\text {loc }}^{s}(b)$ intersect transversally. Now a solution $u$ of (1.2) lies in $W^{1, p}(\Omega \times[-k, k]]$ for each $k$ and $1<p<\infty$ by the regularity theory in [12] and since $h$ is Lipschitz, $h(u)$ is also in $W^{1, p}(\Omega \times[-k, k])$. Hence, by the regularity theory again, we see that $u_{t t}$ is defined and is in $L^{p}(\Omega \times[-k, k])$. Hence, we can easily justify differentiating equation (1.2) for $u$ in $t$ and proving that $u^{\prime}(t)$ is a solution of $\dot{s}=\Delta s+f^{\prime}(u(t)) s$ on $\Omega \times \mathbb{R}$ (remember that $f$ is strictly differentiable at $u$ ). Technically, it is easier to work on a weak form of the equation. Since we have proved that the unstable manifold is a $C^{1}$ manifold (and the unstable manifold is strictly differentiable at appropriate points), we can argue as in [11] to prove that our transversality and related assumptions ensure that the problem $\dot{s}=\Delta s+h^{\prime}(u) s$ on 
$\Omega \times \mathbb{R}, s=0$ on $\partial \Omega \times \mathbb{R}$ and $h \rightarrow 0$ as $|t| \rightarrow \infty$ has up to scalar multiples a unique solution which is $u^{\prime}(t)$ (and transversality is equivalent to this). Now we consider the problem

$$
\begin{array}{ll}
\dot{s}=\Delta s+g(x) s+\omega(t, x) & \text { on } \Omega \times \mathbb{R}, \\
s=0 & \text { on } \partial \Omega \times \mathbb{R},
\end{array}
$$

where $g$ is bounded and $-\Delta-g(x) I$ and the boundary condition is invertible. We let $X$ be $L^{2}(\Omega)$. If $\omega \in C(\mathbb{R}, X) \cap L^{\infty}(\mathbb{R}, X)$, then as in [13], (2.2) has a unique solution in $L^{\infty}(\mathbb{R}, X), \tilde{h}=L \omega$, where

$$
L \tilde{h}(t)=\int_{-\infty}^{t} T(t-s) P_{1} \omega(s) d s-\int_{t}^{\infty} T(s-t) P_{2} \omega(s) d s .
$$

Here $P_{1}$ is the orthogonal projection on $L^{2}(\Omega)$ where the range is spanned by the eigenfunctions corresponding to the eigenfunctions of $-\Delta-g(x) I$ corresponding to positive eigenvalues and $P_{2}=I-P_{1}$. Note that $R\left(P_{2}\right)$ is a finite-dimensional space. Hence, the semigroup $\left.T(t)\right|_{R\left(P_{2}\right)}$ naturally extends to a group. Thus everything makes sense. It is easy to check that $L$ is a bounded linear map of $L^{\infty}(\mathbb{R}, X)$ into itself. Moreover, $L$ maps $C_{0}(\mathbb{R}, X)$ into itself and by standard regularity theorems as in [11], $L$ is a bounded linear map of $L^{\infty}(\mathbb{R}, X)$ into $L^{\infty}\left(\mathbb{R}, X^{\alpha}\right)$ for $0<\alpha<1$. (Hence, we have compactness of $L$ on $L^{\infty}(\mathbb{R}, X)$ when restricted to compact $t$ intervals. Note that the estimates in [12] guarantee a bound for $u_{t}$ in $L^{2}(X \times[-T, T])$ and hence we have compactness in $t$ on bounded $t$ intervals.) Now much as in [13], we can deduce a number of consequences. If $m \in L^{\infty}(X \times \mathbb{R}), m(t, x) \rightarrow m_{ \pm}(x)$ as $t \rightarrow \pm \infty$ uniformly in $x$ and $-\Delta-m_{ \pm}(x) I$ (and the boundary condition is invertible) then the linear operator $\tilde{L}$, defined by

$$
\tilde{L} \tilde{h}=\dot{\tilde{h}}-\Delta \tilde{h}-m \tilde{h}
$$

with the natural domain, is a closed linear operator on $C_{0}(\mathbb{R}, X)$ and is semi-Fredholm of Fredholm index less than infinity. One shows that $\tilde{L} h_{n} \rightarrow 0$ in our space implies that $\left\{h_{n}\right\}$ has a convergent subsequence. (The invertibility for the limit operators as $t \rightarrow \pm \infty$ and the local compactness of $L$ in $t$ plays an essential role here.) Moreover, using the fact that compact perturbations of Fredholm operators do not change their Fredholm index, one proves that this index is determined solely by $m^{+}$and $m^{-}$. To calculate the index of the Fredholm operator, we can easily use compact perturbations to reduce to the case where $m(x, t)$ is $m_{-}(x)$ for $t<-\frac{1}{2}$ and $m_{+}(x)$ for $t>\frac{1}{2}$. Here it is much easier to calculate the index. (The point is that we can semi-explicitly calculate the solutions on $\left[\frac{1}{2}, \infty\right)$ and $\left(-\infty,-\frac{1}{2}\right]$.) Similar arguments appear in [16]. In particular, one can show that the index is +1 if $-\Delta-m_{+} I$ has exactly $n$ negative eigenvalues counting multiplicity, while $-\Delta-m_{-} I$ has exactly $(n-1)$ negative eigenvalues counting multiplicity. Hence, we see that in our original case where our operator $\hat{L} \tilde{h}=\dot{\tilde{h}}-\Delta \tilde{h}-h^{\prime}(\tilde{u}) \tilde{h}$ our operator is Fredholm of index 1. Here $\tilde{u}$ is the 
connecting orbit. Note that $f^{\prime}(\tilde{u}(x, t))$ is defined almost everywhere, is uniformly bounded and $f^{\prime}(\tilde{u}(x, t)) \rightarrow f^{\prime}\left(u_{ \pm}(x)\right)$ as $t \rightarrow \infty$ uniformly in $x$ except on sets of small measure. Since our transversality assumptions imply that this operator has a one-dimensional kernel, it follows that this operator is onto (as a closed linear operator on $C_{0}(\mathbb{R}, X)$ ).

By the second remark after Lemma 3.2 below, we can approximate $h$ uniformly by $C^{1}$ functions $f_{n}$ so that the strictly differentiability holds at $u$ uniformly in $n$. We prove that the conditions for our problem $\dot{u}=\Delta u+h(u)$ continue to hold for the problem $\dot{u}=\Delta u+f_{n}(u)$ for large $n$. This is quite straightforward. The only real problem is to prove that for our perturbed problem there is still a connection close to $\tilde{u}$. To do this, we use our strict differentiability results and apply the implicit function theorem in the space $C_{0}\left((-\infty, \infty), X^{\alpha}\right)=Z$ (noting that strict differentiability rather than $C^{1}$ is all that is needed for the implicit function theorem). Firstly, note that we can translate by a fixed function joining $u_{0}$ and $u_{1}$ to obtain a problem in $C_{0}$. We can write elements of $C_{0}\left((-\infty, \infty), X^{\alpha}\right)$ near $\tilde{u}$ uniquely in the form $\tilde{u}+s \dot{\tilde{u}}+\omega$ where $\omega$ is in a complement $Y$ to span $\dot{\tilde{u}}$ in $C_{0}\left((-\infty, \infty), X^{\alpha}\right)$. Then uniformly in $n$ and for $s$ small, the solutions are of the form $\tilde{u}+s \dot{\tilde{u}}+\omega_{n}(s)$ where $\omega_{n}$ is continuous and is small if $n$ is large. (Note that $f_{n}(y)-h(y)$ is uniformly small and thus $f_{n}(u)-h(u)$ is small in $C_{0}((-\infty, \infty), X)$ if $n$ is large and $u \in Z$ and we use crucially that the derivative is onto.) Thus there is a connection for large $n$. Moreover, the connections near $u_{0}$ form a 1-manifold parameterized by $s$ for all large $n$. Moreover, there is a natural 1-manifold of connections by translating in $t$ (once we know there is one connection). Thus these two manifolds must agree locally and hence globally. Thus for large $n$ there is unique connection near $u_{0}$ (up to translation). The proof of nondegeneracy at $u_{n}^{0}$ (in our sense) is easy because the linearized operator is close to a Fredholm operator which is onto and of index 1.

Since transversality is equivalent to nondegeneracy (as we saw earlier), homotopy invariance of the homotopy index implies that we have reduced our problem to the case where $h$ is $C^{1}$. Note that part of the technicalities in the above argument is really proving that two definitions of transversality are equivalent.

We now use a closely related idea to reduce our problem to a $C^{1}$ finite-dimensional problem. (If we do this, we can easily smooth to reduce the problem to the smooth case and we can then apply the main result in [14].) The main problem is to check that the Conley index condition persists. For large $n$, we consider the problem

$$
\dot{u}=\Delta P_{n} u+P_{n} h\left(P_{n} u\right) \quad \text { on } R\left(P_{n}\right)
$$

where $P_{n}$ was defined earlier. Since the equation $\dot{v}=\Delta v, v=0$ on $\partial \Omega$ has no trivial bounded solutions on $\Omega \times \mathbb{R}$, we can use the product theorem for the Conley index to show that it suffices to consider the problem

$$
\begin{aligned}
& \dot{u}=\Delta u+P_{n} h\left(P_{n} u\right) \\
& u=0 \quad \text { on } \partial \Omega
\end{aligned}
$$


on $X \times \mathbb{R}$. (It is easy to see that the two problems have the same bounded solutions on $\Omega \times \mathbb{R}$.

We need to prove our earlier structure persists for large $n$. This is similar to but somewhat easier than the earlier case. Since $u$ is an approximate solution for large $n$, we again use the implicit function theorem to obtain a connecting orbit for (2.3). (Note that by standard regularity theory $\{\tilde{u}(t) \mid t \in(-\infty, \infty)\}$ lies in a compact set in $X^{\alpha}$ and thus $P_{n} h\left(P_{n} u(t)\right)$ will converge uniformly on $X$ (in $t$ ) as $n \rightarrow \infty$.) We can use very similar but easier arguments to obtain the other properties. The only part that needs a more careful argument is the calculation of the homotopy index for large $n$ on $\tilde{U}$ where $\tilde{U}$ is a small bounded neighbourhood of $\left\{u_{0}, u_{1}, u(t), t \in(-\infty, \infty)\right\}$ in $X^{\alpha}$ which is an isolating block in the sense of [15]. It suffices to prove that any bounded solution $\hat{u}(t)$ on $h$ of

$$
\begin{array}{ll}
\dot{u}=\Delta u+s h(u)+(1-s) P_{n} h(u) & \text { on } \Omega \times \mathbb{R}, \\
u=0 & \text { on } \partial \Omega \times \mathbb{R},
\end{array}
$$

for large $n$ such that $0 \leq s \leq 1$ and $\hat{u}(t)$ lies in the closure of $\tilde{U}$ for all $t$ lies in $\tilde{U}$. Suppose not, that is, suppose that $u_{n}(t)$ are solutions of (2.4) for $s=s_{n}$ such that $u_{n}(t)$ lies in the closure of $\tilde{U}$ for all $t$ and $u_{n}\left(t_{n}\right) \in \partial \tilde{U}$ for some $t_{n}$. By translating we may assume that $u_{n}(0) \in \partial \tilde{U}$. By various local estimates as in [11] we can assume that the $u_{n}$ are uniformly bounded in $X^{\beta}$ and $d u_{n} / d t$ is bounded in $X^{\alpha}$. Thus we can choose a subsequence of the $u_{n}$ such that $u_{n}$ converges uniformly on compact $t$ intervals to $z(t)$ in $X^{\alpha}$. Passing to the limit in (2.4) (using the mild solution formulation), we see that $z(t)$ is a solution of $\dot{u}=\Delta u+h(u)$ on $\Omega \times \mathbb{R}$ such that $z(0) \in \partial \tilde{U}$ and $z(t)$ lies in the closure of $\tilde{U}$ for all $t$. This contradicts $\tilde{U}$ being an isolating block. Hence, we see that the Conley index of the original flow on $\tilde{U}$ is same as that of the finite-dimensional flow and we are finished.

\section{Some technical lemmas}

We prove two technical lemmas needed to complete the proof of Theorem 2.1.

LEMMA 3.1. If $u$ is a nontrivial bounded solution of $\dot{u}=\Delta u+h(u)$ on $\Omega \times \mathbb{R}$ such that $u=0$ on $\partial \Omega \times \mathbb{R}$, then $\{x \mid u(x, t)=0\}$ has measure zero for each $t$.

Proof. Now $u$ satisfies the equation $\dot{u}=\Delta u+b(x, t) u$ where $b$ is bounded and measurable. The result follows from [5, Theorem 4.3(iv)] once we prove that, for any fixed $t$, the map $x \rightarrow u(x, t)$ cannot have a zero of infinite order in $\Omega$. If one exists, it follows from the main result in [1] that $u(x, t) \equiv 0$ on $\Omega$. This is impossible by the forward and backward uniqueness theorems for the parabolic equation. (As before the backwards uniqueness can be found in [2].)

LEMMA 3.2. If $u$ is a nontrivial solution of $\dot{u}=\Delta u+h(u)$ on $\Omega \times[0, T]$, then the map $v \rightarrow S v$ is strictly differentiable at $u$ as a mapping of $C\left([0, T], X^{\alpha}\right)$ to 
itself, where $0<\alpha<1, X=L^{2}(\Omega), X^{\alpha}$ is the fractional power space in the sense of [11] and $S v(t)=\int_{0}^{t} T(t-s) h(v(s)) d s$ for $0 \leq t \leq T$. Here $T(t)$ is the semigroup generated by the Laplacian (with Dirichlet boundary conditions).

PROOF. By standard regularity results for the heat semigroup (see [11, Ch. 1]), it suffices to prove that the map $F(v)=h(v)$ is strictly differentiable at $u$ as a map of $C\left([0, T], X^{\alpha}\right)$ into $C([0, T], X)$. By the Sobolev embedding theorem and interpolation, $X^{\alpha}$ is continuously embedded in $L^{p}(\Omega)$ where $p>2$ (see [11]). Hence, it suffices to prove our result for $F$ considered as a map of $C\left([0, T], L^{p}(\Omega)\right)$ into $C\left([0, T], L^{2}(\Omega)\right)$. If $\delta>0$ is small, we construct a set $Z_{t} \subseteq \Omega$ for each $t$ such that $S_{t}=\{x \in \Omega \mid u(x, t)=0\}$ lies in the interior of $Z_{t}$ and the measure of $Z_{t} \backslash S_{t}$ is at most $\frac{1}{2} \delta$ for each $t$. To prove this, choose a closed neighbourhood $\tilde{Z}$ of $\partial \Omega$ in $\Omega$ such that $\tilde{Z}$ has measure at most $\frac{1}{4} \delta$. For each $t$ we construct a compact neighbourhood $\hat{Z}_{t}$ of $L_{t}=\{x \in \Omega \backslash \operatorname{int} \tilde{Z} \mid u(x, t)=0\}$ in $\Omega$ such that $L_{t}$ lies in the interior of $\hat{Z}_{t}, Z_{t} \cup \partial \Omega$ is compact for each $t$ and $\hat{Z}_{t}$ has measure at most $\frac{1}{4} \delta$. This is possible since $L_{t}$ has zero measure. Now since $u$ is continuous on $\bar{\Omega} \times R$ (by standard regularity theory as in [12]), we see by continuity that for $s$ near $t,\{x \in \Omega \mid u(x, s)=0\}$ lies in the interior of $\hat{Z}_{t} \cup \tilde{Z}$ and $|u(x, s)|$ has a positive lower bound on $\partial\left(\hat{Z}_{t} \cup \tilde{Z}\right) \cap \Omega$ for $s$ near $t$ (where the bound is independent of $s$ ) and $\hat{Z}_{t} \cup \tilde{Z}$ has measure at most $\frac{1}{2} \delta$. By the compactness of $[0, T]$, it follows that we can find $0=t_{1}<t_{2}<\cdots<t_{k}=T$ and $\hat{Z}_{i} \subseteq \Omega$ for $1 \leq i \leq k-1$ such that if $t_{i} \leq t \leq t_{i+1},\{(x, t) \mid u(x, t)=0\} \subseteq \hat{Z}_{i}$, $|u(x, t)|$ has a positive lower bound on $\partial \hat{Z}_{i} \cap \Omega$ and each $\hat{Z}_{i}$ has measure at most $\frac{1}{2} \delta$. Let $\gamma$ be the infimum of the lower bounds and if $s \in C\left([0, T], L^{p}(\Omega)\right)$ has small enough norm, then for each $t,\|s(t)\|_{p}$ is small and hence $\left\{x:|s(t)(x)| \geq \frac{1}{2} \delta\right\}$ has measure at most $\frac{1}{2} \delta$. Thus, for each $t \in[0, T]$,

$$
W_{t}=\left\{x \mid u(x, t)+s_{1}(t)(x), u(x, t)+s_{2}(t)(x) \text { and } u(x, t)\right.
$$

are all nonzero and have the same sign $\}$

has the property that $\Omega \backslash W_{t}$ has measure at most $\frac{1}{2} \delta$ if $s_{1}$ and $s_{2}$ both satisfy the smallness condition. If $t \in[0, T]$ and $x \in W_{t}$, we can simply estimate

$$
\begin{gathered}
A_{t}(x)=h\left(u(x, t)+s_{1}(t)(x)\right)-h\left(u(x, t)+s_{2}(t)(x)\right) \\
-h^{\prime}(u(x, t))\left(s_{1}(t)(x)-s_{2}(t)(x)\right)
\end{gathered}
$$

by the mean value theorem and find it is bounded by $\hat{\varepsilon}\left|s_{1}(t)(x)-s_{2}(t)(x)\right|$. If $t \in$ $[0, T]$ and $x \notin W_{t}$, then $\left|A_{t}(x)\right| \leq K_{1}\left|s_{1}(t)(x)-s_{2}(t)(x)\right|$ by the Lipschitz property of $h$. Hence,

$$
\begin{aligned}
\left\|A_{t}\right\|_{2, \Omega-W_{t}} & \leq K_{1}\left\|s_{1}(t)-s_{2}(t)\right\|_{2, \Omega \backslash W_{t}} \\
& \leq K_{1}\left(m\left(\Omega \backslash W_{t}\right)\right)^{1 / 2-1 / p}\left\|s_{1}(t)-s_{2}(t)\right\|_{p}
\end{aligned}
$$

by Hölder's inequality. Since $m\left(\Omega-W_{t}\right)$ is small for all $t \in[0, T]$ we are in a similar situation to the proof in $[7$, p. 248] and we can easily complete the proof much as there. 


\section{REMARKS.}

(1) We can also handle the case of an interval $(-\infty, \infty)$ provided $u(t)$ approaches a nontrivial solution of the elliptic equation as $t \rightarrow \infty$ and a (necessarily different) nontrivial solution as $t \rightarrow-\infty$. The point is that we can use a single $\hat{Z}_{i}$ for large negative $t$ and a single $\hat{Z}_{i}$ for large positive $t$ where these are determined by two nontrivial solutions of the elliptic equation.

(2) There is a very useful variant of Lemma 3.2. Assume that $g_{n}: R \rightarrow R$ are globally Lipschitz with Lipschitz constant $K$ independent of $n$, and $g_{n}$ is $C^{1}$ for $t \neq 0$ and, given $\alpha>0, g_{n}(t)=0$ if $|t| \geq \alpha$ and $n$ is large. Then it is easy to see by a similar argument that for large $n$ the inequality for strict differentiability at $\tilde{u}$ holds uniformly in $n$. The point is that

$$
\begin{gathered}
g_{n}\left(u(x, t)+h_{1}(x, t)\right)-g_{n}\left(u(x, t)+h_{2}(x, t)\right) \\
-g_{n}^{\prime}(u(x, t))\left(h_{1}(x, t)-h_{2}(x, t)\right)
\end{gathered}
$$

vanishes except on a set $M_{t} \subseteq \Omega$ for each $t$ where $M_{t}$ has small measure uniformly in $t$. Since it is easy to construct $g_{n}$ as above uniformly small such that $f_{n}=h+g_{n}$ is $C^{1}, f_{n}$ is a $C^{1}$ approximation to $h$ such that $f_{n}-h$ is uniformly small and such that Lemma 3.2 holds for $f_{n}$ for large $n$ with the neighbourhood independent of $n$. This is crucial in Section 2.

\section{References}

[1] G. Alessandrini and S. Vessella, 'Remark on the strong unique continuation property for parabolic operators', Proc. Amer. Math. Soc. 132 (2004), 499-501 (electronic).

[2] C. Bardos and L. Tartar, 'Sur l'unicité rétrograde des équations paraboliques et quelques questions voisines', Arch. Ration. Mech. Anal. 50 (1973), 10-25.

[3] L. A. Caffarelli and A. Friedman, 'Partial regularity of the zero-set of solutions of linear and superlinear elliptic equations', J. Differential Equations 60 (1985), 420-433.

[4] H. Cartan, Calcul Différentiel (Hermann, Paris, 1967).

[5] X.-Y. Chen, 'On the scaling limits at zeros of solutions of parabolic equations', J. Differential Equations 147 (1998), 355-382.

[6] C. Conley, Isolated Invariant Sets and the Morse Index, CBMS Regional Conference Series in Mathematics, 38 (American Mathematical Society, Providence, RI, 1978).

[7] E. N. Dancer, 'On positive solutions of some pairs of differential equations. II', J. Differential Equations 60 (1985), 236-258.

[8] - 'On connecting orbits for competing species equations with large interactions', Topol. Methods Nonlinear Anal. 24 (2004), 1-19.

[9] E. N. Dancer and Y. H. Du, 'Competing species equations with diffusion, large interactions, and jumping nonlinearities', J. Differential Equations 114 (1994), 434-475.

[10] E. N. Dancer and Z. Zhang, 'Dynamics of Lotka-Volterra competition systems with large interaction', J. Differential Equations 182 (2002), 470-489.

[11] D. Henry, Geometric Theory of Semilinear Parabolic Equations, Lecture Notes in Mathematics, 840 (Springer, Berlin, 1981).

[12] G. M. Lieberman, Second Order Parabolic Differential Equations (World Scientific, River Edge, NJ, 1996).

[13] A. Lunardi, Analytic Semigroups and Optimal Regularity in Parabolic Problems, Progress in Nonlinear Differential Equations and their Applications, 16 (Birkhäuser, Basel, 1995). 
[14] C. McCord, 'The connection map for attractor-repeller pairs', Trans. Amer. Math. Soc. 307 (1988), 195-203.

[15] K. P. Rybakowski, The Homotopy Index and Partial Differential Equations (Springer, Berlin, 1987).

[16] A. Vol'pert and V. Vol'pert, 'The Fredholm property of elliptic operators in unbounded domains', Tr. Mosk. Mat. Obs. 67 (2006), 148-227.

E. N. DANCER, School of Mathematics and Statistics, University of Sydney, NSW 2006, Australia

e-mail: E.Dancer@maths.usyd.edu.au 